\title{
The Structure of High Redshift Galactic Halos
}

\author{
Sara L. Ellison \\ P. Universidad Catolica de Chile, Casilla 306, Santiago 22, Chile; \\ European Southern Observatory, Casilla 19001, Santiago 19, Chile; \\ University of Victoria, 3800 Finnerty Rd., Victoria, BC, V8P 1A1, \\ Canada.
}

Rodrigo Ibata

Observatoire de Strasbourg, 11, rue de l'Université, F-67000, Strasbourg, France

\author{
Max Pettini \\ Institute of Astronomy, Madingley Rd, Cambridge, CB3 OHA, U.K. \\ Geraint F. Lewis \\ Institute of Astronomy, School of Physics, A 29, University of Sydney, \\ NSW 2006, Australia
}

\author{
Bastien Aracil, Patrick Petitjean \\ Institut d'Astrophysique de Paris - CNRS, 98bis Boulevard Arago, \\ F-75014 Paris, France \\ R. Srianand
}

IUCAA, Post Bag 4, Ganeshkhind, Pune 411 007, India

\begin{abstract}
.
Observations of multiple or lensed QSOs at high redshift can be used to probe transverse structure in intervening absorption systems. Here we present results obtained from STIS spectroscopy of the unique triply imaged QSO APM $08279+5255$ at $z_{\mathrm{em}}=3.9$ and study the coherence scales of intervening low and high ionization absorbers.
\end{abstract}

\section{Introduction}

As we have heard throughout this meeting, absorption line spectroscopy is a powerful and accurate technique for determining abundances in the interstellar and intergalactic media. The advantages of this technique become even more pronounced for applications in the high redshift universe where the familiar UV transitions used to determine gas phase abundances are shifted into the optical. In this regime these transitions can be studied with efficient CCD detectors mounted on large ground-based telescopes. By exploiting these advan- 


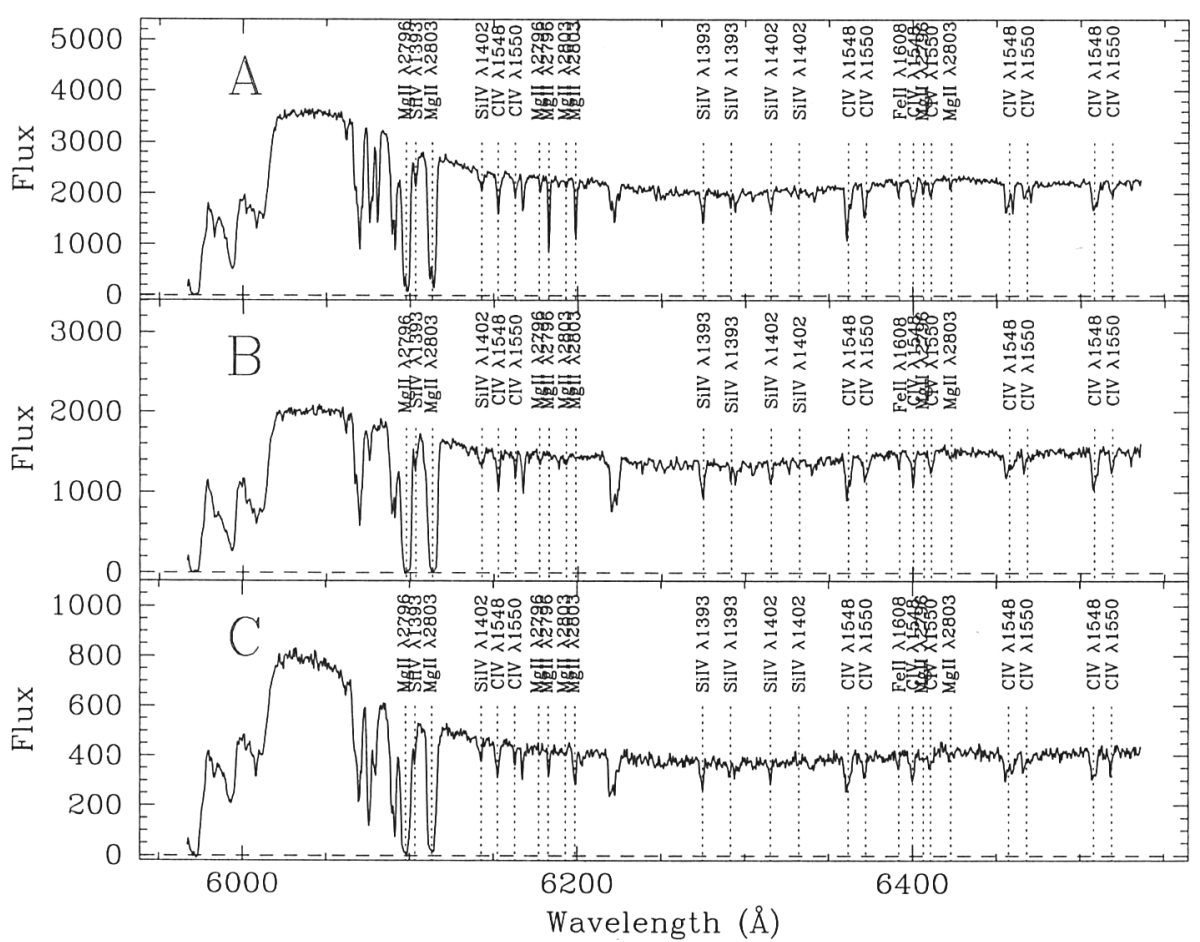

Figure 1. Example of the extracted STIS spectra for the 3 components of the triply imaged QSO with intervening absorbers labelled.

tages, enormous progress has been in the last decade in determining the metal abundances of high redshift absorption systems with accuracies that rival those possible for Galactic stars. Given the impressive bulk of abundance data for a range of chemical elements, it is somewhat ironic that other (perhaps more fundamental) properties of absorption galaxies such as size, luminosity and morphology remain relatively unconstrained, with information available for only a handful of low redshift systems.

One technique that has been used to expand upon the single dimension studies of QSO absorbers is to use multiple, but closely spaced lines of sight, either via gravitational lensing or chance alignments of quasars. The multiple lines of sight (LOS) will then intersect intervening galaxies at different transverse locations, offering the opportunity to probe the absorber on parsec to kiloparsec scales (e.g. Rauch 2002). In order to improve our knowledge of the sizes and internal velocity structure of high redshift absorbing galaxies, we have undertaken a program with HST to obtain spatially resolved spectra of the lensed QSO APM $08279+5255\left(z_{\mathrm{em}}=3.9\right)$, a unique triply imaged QSO (Ibata et al. 1999; Lewis et al. 2002) with image separations ranging between 0.15 and 0.38 arcsec. This sightline is particularly rich with absorption systems over a large redshift range (Ellison et al. 1999a,b) and therefore offers an exceptional opportunity to study the intervening gas on a range of transverse scales. 


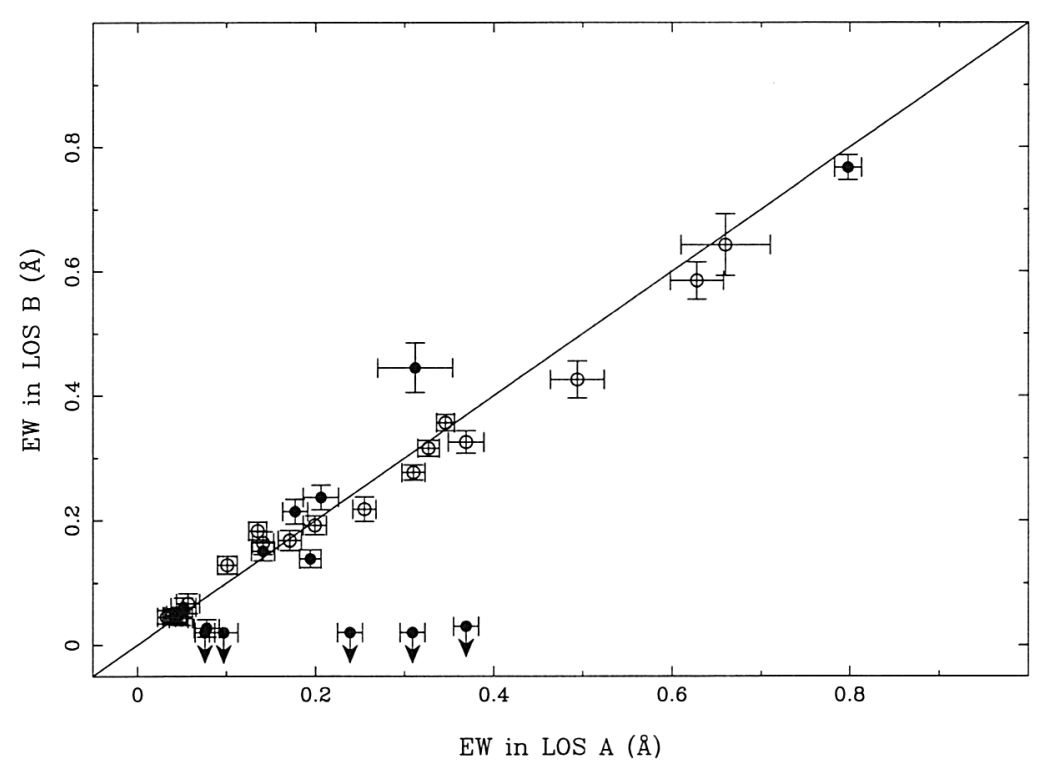

Figure 2. Rest frame EW comparison for absorption systems in two of the components (A and B) of the lensed QSO APM 08279+5255. High ionization systems are plotted with open points, low ionization systems are filled.

We assume the 'consensus' cosmology of $\Omega_{M}=0.3, \Omega_{\Lambda}=0.7, H_{0}=$ $70 \mathrm{~km} \mathrm{~s}^{-1} \mathrm{Mpc}^{-1}$ throughout and convert literature values to this scale when making comparisons. We also assume that the redshift of the lensing galaxy is $z_{\text {lens }}=1.062$.

\section{An Overview of the Data}

We have obtained spatially resolved spectra of the 3 components of APM 08279 +5255 with STIS on board the HST. A total of 25 orbits was divided between 5 wavelength settings to achieve a total coverage of $\sim 6000$ to $\sim 8600 \AA$. The spectral resolution is $1.6 \AA \mathrm{FWHM}$ and the typical $\mathrm{S} / \mathrm{N}$ per pixel ranges from 60 to 20. In Figure 1 we plot an example of the extracted STIS spectra with absorption systems labelled. Full details of the data acquisition and reduction, as well as measurements of absorption line equivalent widths (EWs) can be found in Ellison et al. (2003).

\section{Comparison of Low and High Ionization Absorbers}

In Figure 2 we show a comparison of EWs for low ( $\mathrm{Mg}$ II and Fe II) and high (C IV and Si IV) ionization absorbers in 2 of the 3 LOS, labelled A and B (see Ibata et al. 1999 for NICMOS imaging of the three closely spaced components). The striking result of this plot is that the high ionization systems trace each other relatively closely in terms of line strength, whereas there can be marked 


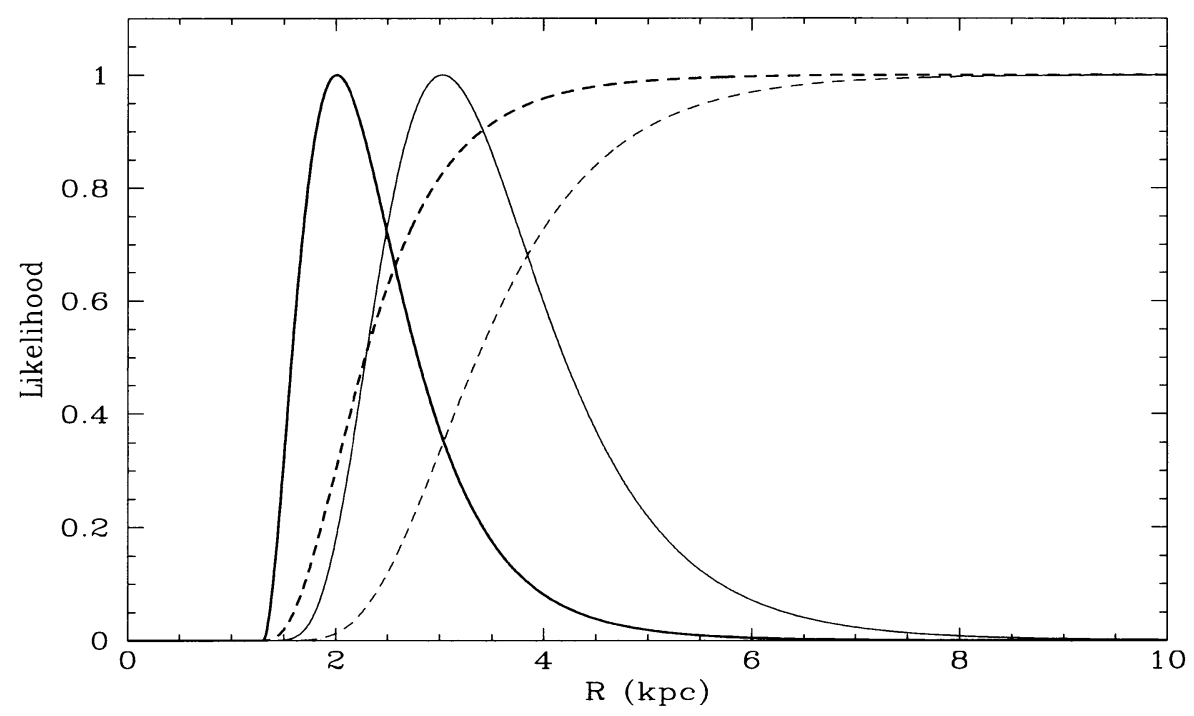

Figure 3. Maximum likelihood distribution (thin solid line) and cumulative distribution (dashed line) for spherical halos normalized to the peak value. The most likely coherence radius is found to be $\mathrm{R}=3.0$ $h_{70}^{-1} \mathrm{kpc}$ with $95 \%$ confidence limits of 2.1 and $6.2 h_{70}^{-1} \mathrm{kpc}$. The bold lines show the results obtained if we restrict ourselves to weak $(\mathrm{EW}<0.3 \AA) \mathrm{Mg}$ II systems only, for which we deduce a most likely scale of $R=2.0 h_{70}^{-1} \mathrm{kpc}$ with $95 \%$ confidence limits of 1.5 and $4.4 h_{70}^{-1}$ kpc.

differences in the low ionization systems. However, one should bear in mind that the redshifts, and therefore transverse separations, are different for these systems; C IV absorbers are observed with typical separations of several hundred pc, whereas Mg II systems probe scales of a few kpc. Nonetheless, these results confirm earlier work by Rauch et al. $(1999,2000,2001)$ that cooler, low ionization gas is clumped on smaller scales than the warmer, more highly ionized component.

\section{Coherence Scales of Absorbers}

C IV absorbers have been studied in lensed sightlines on a range of scales that confirm coherence over $\sim 100 h_{70}^{-1} \mathrm{kpc}$ (e.g. Petitjean et al. 1998; Lopez et al. 2000). The transverse separations towards APM $08279+5255$ are considerably smaller than this, and, as is evident from Figure 2, a high degree of coherence is seen at $z \sim 3$ on scales of $100 \mathrm{~s}$ of pc. However, little work has been done previously on the low ionization systems due to a paucity of statistics. For the first time, we have a sufficient number of $\mathrm{Mg}$ II systems to attempt a maximum likelihood estimate of coherence scale based on the number of coincidences and anti-coincidences in pairs of LOS using the technique of McGill (1990). For the full sample of $\mathrm{Mg}$ II absorbers we find a most likely coherence radius of 

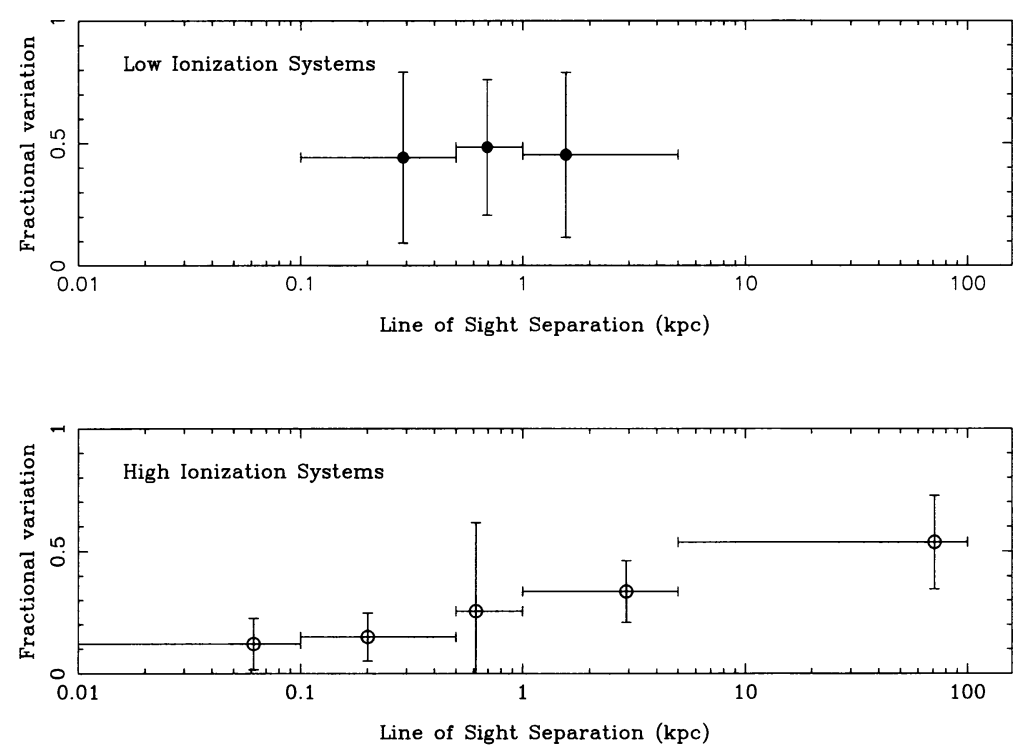

Figure 4. Fractional EW variations for low and high ionization systems from this work and the literature (Lopez et al. 2000; Rauch et al. 2001; Rauch et al. 2002; Churchill et al. 2003).

$R=3.0 h_{70}^{-1} \mathrm{kpc}$, with $95 \%$ confidence limits of 2.1 and $6.2 \mathrm{kpc}$ (Figure 3 ). This is apparently at odds with the size of $\mathrm{Mg}$ II halos, $\sim 50 \mathrm{kpc}$, inferred by Steidel, Dickinson, \& Persson (1994) from the number density of absorbers and galaxy impact parameters. However, we can divide our sample of $\mathrm{Mg}$ II absorbers into 'strong' ( $\mathrm{EW} \geq 0.3 \AA$, the typical $\mathrm{EW}$ of most absorption line system surveys) and weak (EW $<0.3 \AA$ ) subsets. We can only place a lower limit of $R>1.4 h_{70}^{-1} \mathrm{kpc}$ on the strong absorbers because all systems are seen in all LOS. For weak systems, we infer a most likely coherence radius of $2.0 h_{70}^{-1} \mathrm{kpc}$ with $95 \%$ confidence limits of 1.5 and $4.4 \mathrm{kpc}$ (see Figure 3). This could be explained if the filling factor of the low ionization gas decreases towards the outer part of the galaxy, leading to a smaller coherence scale for low EW absorbers. This scenario seems to be qualitatively supported by the observations of Churchill et al. (2000) that impact parameter is anti-correlated with $N(\mathrm{Mg} \mathrm{II})$.

We can also examine whether absorption systems exhibit larger transverse variations as a function of LOS separation. In Figure 4 we have plotted the mean fractional variation in EW for absorption systems in this study and for data compiled from the literature as a function of transverse separation. It can be seen that for the low ionization systems the mean variation is the same for all separations within the large scatter. This is probably due to the fact that individual components cannot be traced over more than a few hundred pc (Rauch et al. 2002). The high ionization systems, however, show a clear trend for larger variations with increasing LOS separation.

These results are qualitatively similar to observations of the Galaxy, where cold gas traced by Na I shows fine spatial structure on AU scales (e.g. Lauroesch, 
Meyer \& Blades 2000). Slightly warmer, but still predominantly neutral, gas marked by Ca II (which is comparable to the phase traced by $\mathrm{Mg}$ II and Fe II studied here) also shows small scale variations, but is less clumpy than the cold gas (e.g. Redfield \& Linsky 2002). The warm ionized component, traced for example by C IV, is much more smoothly distributed and shows far less variation (Savage, Sembach \& Lu 1997).

These results show the power of multiple LOS spectroscopy for providing information on the spatial extent and kinematics of intervening galaxies which cannot be obtained by other means.

Acknowledgments. SLE is very grateful to the IAU for a generous travel grant and to the SOC and LOC for their help in facilitating attendance at this enjoyable symposium.

\section{References}

Churchill, C. W., Mellon, R. R., Charlton, J. C., Jannuzi, B. T., Kirhakos, S., Steidel, C. C., Schneider, D. P., 2000, ApJ, 543, 577

Churchill, C. W., Mellon, R. R., Charlton, J. C., Vogt, S., 2003, ApJ, 593, 203

Ellison, S. L., Ibata, R., Pettini, M., Lewis, G., Aracil, B., Petitjean, P., Srianand, R., 2003, A\&A, submitted.

Ellison, S. L., Lewis, G. F., Pettini, M., Chaffee, F. H., Irwin, M. J., 1999a, ApJ, 520, 456

Ellison, S. L., Lewis, G. F., Pettini, M., Sargent, W. L. W., Chaffee, F. H., Foltz, C. B., Rauch, M., Irwin M. J., 1999b, PASP, 111, 946

Ibata, R., Lewis, G. F., Irwin, M. J., Lehar, J., Totten, E. J., 1999, AJ, 118, 1922

Lauroesch, J. T., Meyer, D. M., \& Blades, J. C., 2000, ApJ, 543, L43

Lewis, G. F., Ibata, R., A., Ellison, S. L., Aracil, B., Petitjean, P., Pettini, M., Srianand, R., 2002, MNRAS, 334, L7

Lopez, S., Hagen, H.-J., Reimers, D., 2000, A\&A, 357, 37

McGill, C., 1990, MNRAS, 242, 544

Petitjean, P., Surdej, J., Smette, A., Shaver, P., Mücket, J., Remy, M., 1998, A\&A, 334, L45

Rauch, M., 2002, ASP Conference Proceedings, 'Extragalactic Gas at Low Redshift', Eds, J. Mulchaey, J. Stocke, 254, 140

Rauch, M., Sargent, W. L. W., \& Barlow, T. A., 1999, ApJ, 515, 500

Rauch, M., Sargent, W. L. W., \& Barlow, T. A., 2001, ApJ, 554, 823

Rauch, M., Sargent, W. L. W., Barlow, T. A., Simcoe, R. A., 2002, ApJ, 576, 45

Redfield, S. \& Linsky, J. L., 2002, ApJS, 139, 439

Savage, B. D., Sembach, K. R., \& Lu, L., 1997, AJ, 113, 6

Steidel, C. C., Dickinson, M., \& Persson, E. 1994, ApJ, 437, L35 